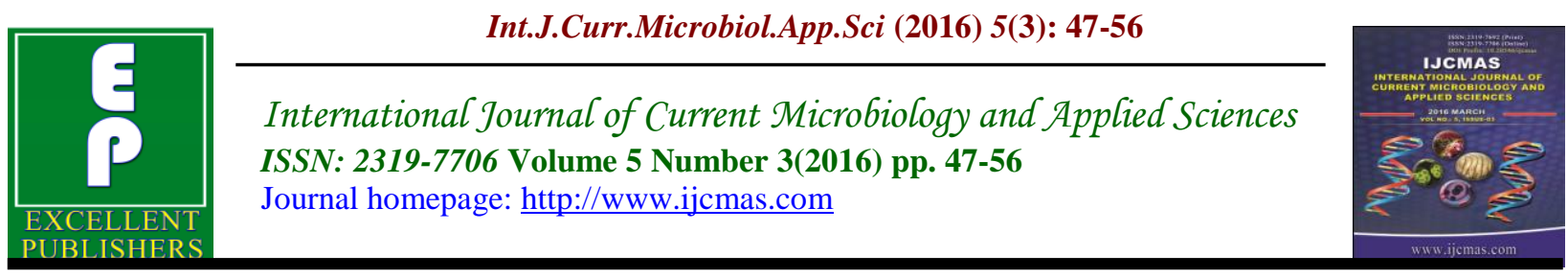

Original Research Article

http://dx.doi.org/10.20546/ijcmas.2016.503.007

\title{
Phenotypic Detection of Biofilms in Candida Species Isolated from Various Clinical Samples
}

\begin{abstract}
M. Bansal*, S.A. Samant, S. Singh and A. Talukdar
Department of Microbiology, MGM Medical College and Hospital, Kamothe, Navi Mumbai, India

*Corresponding author

A B S T R A C T

Keywords

Candida biofilm,

Phenotypic

detection,

Antifungal,

Plant oils.

\section{Article Info}

Accepted:

07 February 2016

Available Online:

10, March 2016

Since biofilms have been considered as a virulence factor contributing to the Candidal infection, a reliable method for their diagnosis is necessary. In this study, we screened 100 clinical isolates of Candida spp. by Congo Red Agar Method, Test Tube method and Micro titer Plate Method. Biofilms have great significance in public health, because biofilm-associated Candida exhibit dramatically decreased susceptibility to antimicrobial agents. This susceptibility may be intrinsic (as a natural outcome of growth in the biofilm) or acquired (due to transfer of extra chromosomal elements to susceptible organisms in the biofilm). It is likely that biofilms evade anti microbial challenges by multiple mechanisms. In this study $57 \%$ of the clinical Candida isolates showed Biofilm production by Test tube method. It was found that $C$. albicans had a little lower percentage of biofilm positivity $(4 / 9=44.4 \%)$ as compared to non-albicans Candida spp. $(53 / 91=$ $58.2 \%$ ). Antifungal efficacy of Coconut oil and Eucalyptus oil was also tested in this study against all Candida isolates. Eucalyptus oil was observed to be a better antifungal agent than Coconut oil in the present study. When coconut oil was tested against all Candida albicans isolates, the sensitivity of biofilm non producers was higher in comparison to biofilm producers.
\end{abstract}

\section{Introduction}

Candida is a genus of yeasts and is currently the most common cause of fungal infections worldwide (Manolakaki et al., 2002). Many species are harmless commensals or endosymbionts of hosts including humans; however, when mucosal barriers are disrupted or the immune system is compromised they can invade and cause disease (Kourkoumpetis et al., 2011). Candida is almost universal in low numbers on healthy adult skin and C. albicans is part of the normal flora of the mucous membranes of the respiratory, gastrointestinal, and female genital tracts. Fungi (mainly $C$. albicans) are the third leading cause of catheter-related infections, representing the second highest colonization-to-infection rate and the overall highest crude mortality (Crump J.A. and Collignon P.J., 2000). 
The formation of Candida biofilms carries important clinical repercussions because of their increased resistance to antifungal therapy and the ability of cells within biofilms to withstand host immune defenses. Also, biofilm formation on medical devices can negatively impact the host by causing the failure of the device and by serving as a reservoir or source for future continuing infections (Douglas L.J., 2002; Maki D.G. et al., 1997).

The net effect is that Candida biofilms adversely impact the health of these patients with increasing frequency and severity and with soaring economic sequel. Hence detection of biofilms becomes necessary.

Various methods are currently used in medical areas for the detection of biofilm production, such as the tube adherence test described by Christensen et al., (Christensen G.D. et al., 1982) and the Congo Red Agar (CRA) method described by Freeman et al., (Freeman D.J. et al., 1989) and quantitative methods such as the Tissue Culture Plate (TCP) assay described by Christensen et al., (Christensen G.D. et al., 1985 ) are used in routine laboratories. In this study, phenotypic detection of biofilms in Candida species isolated from various clinical samples was done by three different methods in this project.

Yeast infections are typically treated with antifungal medications called antimycotics. While these types of medications are effective for many, some may experience side effects and a rebound infection (Ogbolu D.O. et al., 2007).

To avoid side effects, one can use naturally occurring antifungals. Various researches have been done to detect any such natural product. One new research shows that eucalyptus oil and coconut oil has powerful anti-fungal properties and it may be an effective treatment (Ogbolu D.O. et al., 2007).

This antifungal efficacy of eucalyptus oil and coconut oil on the clinical Candida isolates was also tested through this project.

The present study aims at:

1) Isolation of Candida spp. From various specimens

2) Detection of biofilm forming capacity of these isolates by phenotypic methods

3) Assessment of antifungal efficacy of plant oils on pathogenic Candida isolates.

\section{Materials and Methods}

This study was conducted over a period of one year (December 2013 to December 2014) in the Department of Microbiology, MGM Medical College \& Hospital, Kamothe, Navi Mumbai. A total of 100 isolates of Candida recovered from clinical specimens like urine, pus, blood, body fluids, sputum, etc were included in the study. The clinical isolates recovered from both outdoor and indoor patients, irrespective of their age and gender, were identified by standard microbiological procedures.

\section{Phenotypic Determination of Biofilm Formation}

Congo Red Agar Method (CRA) (Dag I. et al., 2010). The medium is composed of BHI $(37 \mathrm{gms} / \mathrm{L})$, glucose $(80 \mathrm{gms} / \mathrm{L})$, agar no. 1 $(10 \mathrm{gms} / \mathrm{L})$ and congo red stain $(0.8 \mathrm{gms} / \mathrm{L})$. Congo red was prepared as concentrated aqueous solution and autoclaved at $121^{\circ} \mathrm{C}$ for $15 \mathrm{~min}$, separately from other medium constituents and was then added when the agar had cooled to $55^{\circ} \mathrm{C}$ (Freeman et al., 1989). 
Plates were inoculated and incubated aerobically for 24 to 48 hours at $37^{\circ} \mathrm{C}$. Positive result was indicated by dark red colonies.

Tube Method (TM) (Dag I. et al., 2010)

A loopful of organisms from the surface of SDA plate was inoculated into polystyrene tube containing $10 \mathrm{ml}$ of SDB supplemented with glucose. The tubes were incubated at $35^{\circ} \mathrm{C}$ for 48 hours. After incubation, the broth in tubes was aspirated gently. The tubes were washed once with distilled water. Then the tubes were stained with $1 \%$ safranin after media and yeast cells were discarded.

\section{Microtiter Plate Method (MTP)} (Dhanasekaran D. et al., 2014)

Individual wells of sterile, polystyrene, 96 well-flat bottom microtitre plates were filled with $100 \mu \mathrm{l}$ aliquots of the cell suspension. The microlitre plates were incubated for $72 \mathrm{~h}$ at $37^{\circ} \mathrm{C}$. After incubation content of each well was gently removed by tapping the plates. Plates were stained with crystal violet $(0.1 \% \mathrm{w} / \mathrm{v})$.

Excess stain was rinsed off by thorough washing with deionized water and plates were kept for drying. Optical density (OD) of stained adherent Candida biofilm was determined with ELISA reader at wavelength of $492 \mathrm{~nm}$. These OD values were considered as an index of Candida adhering to surface and forming biofilms.

\section{Antifungal Efficacy Testing of Eucalyptus Oil and Coconut Oil}

Preparation of Impregnated Paper Discs (Epka O.D. and Ebana R.U.B., 1996)

The commercially available extracts of Eucalyptus oil and coconut oil were used. A
$0.04 \mathrm{ml}$ of $100 \%$ concentration of the Eucalyptus oil and coconut oil extracts was impregnated into the discs.

The impregnated discs were left to dry for 24 hours in the incubator at $37^{\circ} \mathrm{C}$. After drying, the discs were transferred back into the sterile container and stored in the refrigerator at $4^{\circ} \mathrm{C}$.

Antimicrobial Sensitivity Test (Brauer, A.W. et al., 1996)

Antimicrobial experiments on Candida albicans were carried out on SDA plates. The test strain were streaked on the test plates using sterile wire loop and allowed to dry for 15 minutes. Sterile impregnated discs were applied onto the inoculated plates. The plates were then incubated at $37^{\circ} \mathrm{C}$ for $24-48$ hours. Zones of inhibition were assessed after the period of incubation. (Mean size-5mm).

\section{Results and Discussion}

In the present study, 100 Candida spp. isolated from clinical samples namely, urine, vaginal swab, sputum, pus, blood, etc. As depicted in Table 1, the highest number of Candida spp. were isolated from urine (41), followed by vaginal swab (26). The least number was found in throat swab (1), central line tip (1) and ear swab (1). It also shows the prevalence of $C$. albicans and nonalbicans Candida in these samples. Number of non-albicans Candida was much higher (91\%) as compared to C. albicans (9\%). This underlines the importance of pathogenic role of non-albicans Candida. Similar findings have also been reported by Golia et al., (Golia S)

In the present study, all the clinical isolates of Candida were tested by three in vitro screening tests for biofilm production, namely Congo Red Agar, Test Tube method 
and Microtiter Plate Method.

It is known that, Congo red has interaction with various polysaccharides; however it shows high affinity to chitin and glucan (Roncero and Duran, 1985). Congo red not only binds to the carbohydrates of extracellular matrix (ECM) generated by the Candida but also to chitin and glucan present in the cell wall. Therefore, the interaction of Congo red with extracellular matrix and cell wall composition could limit its use in the evaluation of fungal Biofilm formation. Hence CRA test cannot be recommended as a general screening test for Biofilm formation of Candida spp.

Microtiter plate procedure was used according to well-established protocols and can be modified for various Biofilm formation assays. However, because the polysterene plates may not reflect exactly the ability to form Biofilm in vivo, clinical decisions must be given carefully.

Test tube method is fast, reliable, and reproducible method. Cerikcioglu et al (2004) evaluated biofilm production ability of seven Candida isolates with visual tube method and compared their results with the transmission electron microscope (TEM). They suggested that the use of Tube method was simple and reliable for early detection of Biofilm production and it correlated well with TEM. Gokce et al. (2007) studied biofilm production ability of 99 Candida strains isolated from blood cultures using both Tube method and spectrophotometric methods. Comparison of both methods displayed strong correlation. Similarly, Oliveira and Cunha stated that the tube method was reliable for determining of Biofilm production in routine use. (Oliveira and Cunha, 2010)

Also the findings of present study show that it is the most sensitive method for detection

of Biofilm in Candida as it could detect biofilm production in maximum number of isolates $(57 \%)$.

As shown in Table 2 (Figure 2), out of the three phenotypic methods used, the biofilm production was detected in $32 \%$ Candida isolates by CRA method, $34 \%$ by MTP method and $57 \%$ by Test Tube method. Thus, our data indicates that the Test Tube method is a more sensitive method for determining biofilm formation of clinical Candida isolates. Also this method is reliable, cheap and easy to use and does not require any specialized instruments or ingredients. Hence, this method was taken as the standard method for further inferences in this study.

In this study $57 \%$ of the clinical Candida isolates showed Biofilm production by Test tube method. This can be considered to be an important finding because of the fact that biofilm production is a special feature of pathogenicity in Candida spp and more than $50 \%$ isolates have the capacity to produce biofilm.

In Table 3, prevalence of biofilm production in Candida spp. in various clinical samples (Test tube method) has been discussed. Out of total 100 Candida isolates, 41 were from urine. 25 out of 41 urine isolates i.e. $60.9 \%$ isolates showed Biofilm production. This is followed by vaginal swab samples that showed $46.1 \%$ Biofilm production (12/26). Out of 11 sputum samples, 5 were positive for Biofilm i.e. $45.4 \%$. The other samples like pus (6), blood (5), stool (3), suction tip (3), and Foley's tip (2) were less in number. We also obtained 1 isolate each of Candida from Central line tip, throat swab and Ear swab. All these three were positive for Biofilm production. Suction tip and central 
line tip has shown $100 \%$ Biofilm production indicating prevalence of Biofilm in medical devices. But, the data size in this study is too small to get a significant conclusion, hence requires in depth study on Biofilm producing Candida from these specimens.

As interpreted in Table 4 and 5, it was found that $C$. albicans had a little lower percentage of biofilm positivity $(4 / 9=44.4 \%)$ as compared to non-albicans Candida spp. $(53 / 91=58.2 \%)$. With this result, we can infer that biofilm production is prevalent both in $C$. albicans and non-albicans Candida spp. This finding co-relates with the studies of other workers like Tumbarello et al., 2007; Shin et al., 2002; Vinitha and Ballal, 2007. But, it is in contrast with the findings of Ilknur Dag et al., 2010 who has claimed that Candida albicans had slightly more percentage of Biofilm positivity $(39.3 \%)$ with respect to non-albicans Candida spp. (37.7\%)

Table.1 Incidence of Candida spp. in Clinical Samples

\begin{tabular}{|c|c|c|c|c|}
\hline $\begin{array}{c}\text { Serial } \\
\text { No. }\end{array}$ & Sample & $\begin{array}{c}\text { No. of clinical Candida } \\
\text { albicans isolates }\end{array}$ & $\begin{array}{c}\text { No. of clinical non- } \\
\text { albicans Candida isolates }\end{array}$ & Total \\
\hline 1 & Urine & 2 & 39 & 41 \\
\hline 2 & Vaginal swab & 3 & 23 & 26 \\
\hline 3 & Sputum & 1 & 10 & 11 \\
\hline 4 & Pus & 1 & 5 & 6 \\
\hline 5 & Blood & - & 5 & 3 \\
\hline 6 & Stool & 1 & 2 & 3 \\
\hline 7 & Suction tip & - & 2 & 2 \\
\hline 8 & Foley's tip & - & 1 & 1 \\
\hline 9 & Central line tip & - & - & 1 \\
\hline 10 & Throat swab & 1 & 1 & 100 \\
\hline 11 & Ear swab & - & 91 & \\
\hline & Total & 09 & & \\
\hline
\end{tabular}

Table.4 Biofilm Producers in Candida Albicans

\begin{tabular}{|c|c|c|c|c|c|}
\hline Specimen & $\begin{array}{c}\text { No. of Candida } \\
\text { albicans isolates }\end{array}$ & Biofilm (+) & Biofilm (+) \% & Biofilm (-) & Biofilm (-) \% \\
\hline 100 & 9 & 4 & 44.4 & 5 & 55.6 \\
\hline
\end{tabular}

Table.5 Biofilm Producers in Non Albicans Candida

\begin{tabular}{|c|c|c|c|c|c|}
\hline Specimen & $\begin{array}{c}\text { No. of non } \\
\text { albicans Candida } \\
\text { isolates }\end{array}$ & Biofilm (+) & Biofilm (+) \% & Biofilm (-) & Biofilm (-) \% \\
\hline 100 & 91 & 53 & 58.2 & 38 & 41.8 \\
\hline
\end{tabular}


Table.6 Effect of Coconut Oil on Candida albicans

\begin{tabular}{|c|c|c|c|c|}
\hline Specimen & $\begin{array}{c}\text { Sensitive to } \\
\text { Coconut Oil }\end{array}$ & Sensitive & $\begin{array}{c}\text { Resistant to } \\
\text { Coconut Oil }\end{array}$ & Resistant \\
\hline Biofilm producers & 1 & $25 \%$ & 3 & $75 \%$ \\
\hline Biofilm non producers & 2 & $40 \%$ & 3 & $60 \%$ \\
\hline
\end{tabular}

Table.7 Effect of Eucalyptus Oil on Candida albicans

\begin{tabular}{|c|c|c|c|c|}
\hline Specimen & $\begin{array}{c}\text { Sensitive to } \\
\text { Eucalyptus Oil }\end{array}$ & Sensitive & $\begin{array}{c}\text { Resistant to } \\
\text { Eucalyptus Oil }\end{array}$ & Resistant \\
\hline Biofilm producers & 2 & $50 \%$ & 2 & $50 \%$ \\
\hline Biofilm non producers & 5 & $100 \%$ & 0 & - \\
\hline
\end{tabular}

Table.8 Effect of Coconut Oil on Non albicans Candida

\begin{tabular}{|c|c|c|c|c|}
\hline Specimen & $\begin{array}{c}\text { Sensitive to } \\
\text { Coconut Oil }\end{array}$ & Sensitive & $\begin{array}{c}\text { Resistant to } \\
\text { Coconut Oil }\end{array}$ & Resistant \\
\hline Biofilm producers & 8 & $15.1 \%$ & 45 & $84.9 \%$ \\
\hline Biofilm non producers & 12 & $31.6 \%$ & 26 & $68.4 \%$ \\
\hline
\end{tabular}

Table.9 Effect of Eucalyptus Oil on Non albicans Candida

\begin{tabular}{|c|c|c|c|c|}
\hline Specimen & $\begin{array}{c}\text { Sensitive to } \\
\text { Eucalyptus Oil }\end{array}$ & Sensitive & $\begin{array}{c}\text { Resistant to } \\
\text { Eucalyptus Oil }\end{array}$ & Resistant \\
\hline Biofilm producers & 21 & $39.6 \%$ & 32 & $60.4 \%$ \\
\hline Biofilm non producers & 28 & $73.7 \%$ & 10 & $26.3 \%$ \\
\hline
\end{tabular}

Figure.2 Comparison of the Three Methods of Biofilm Detection in Candida Spp

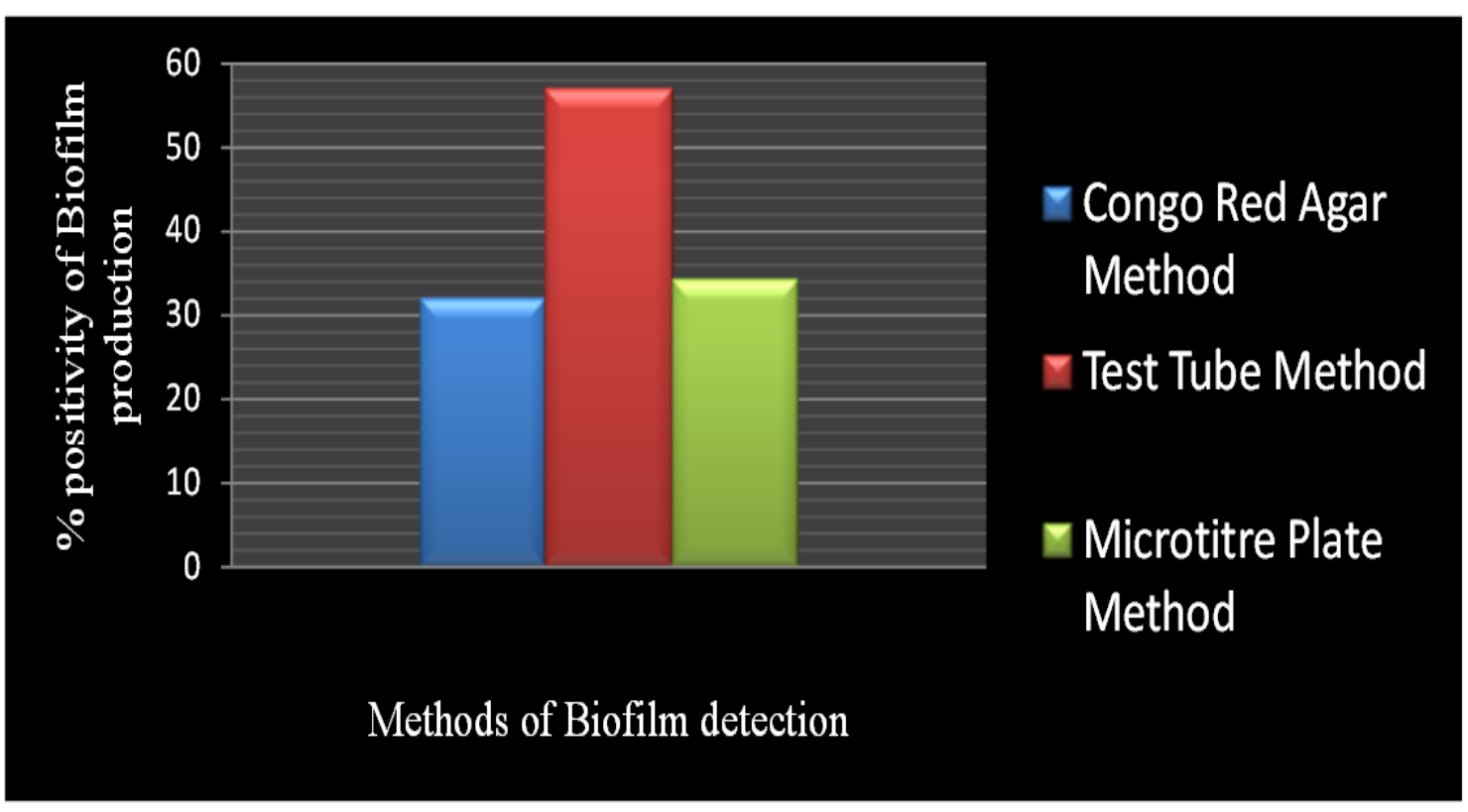


Figure.3 Prevalence of Biofilm Production in Candia spp. in Various Clinical Specimens (Test Tube Method)
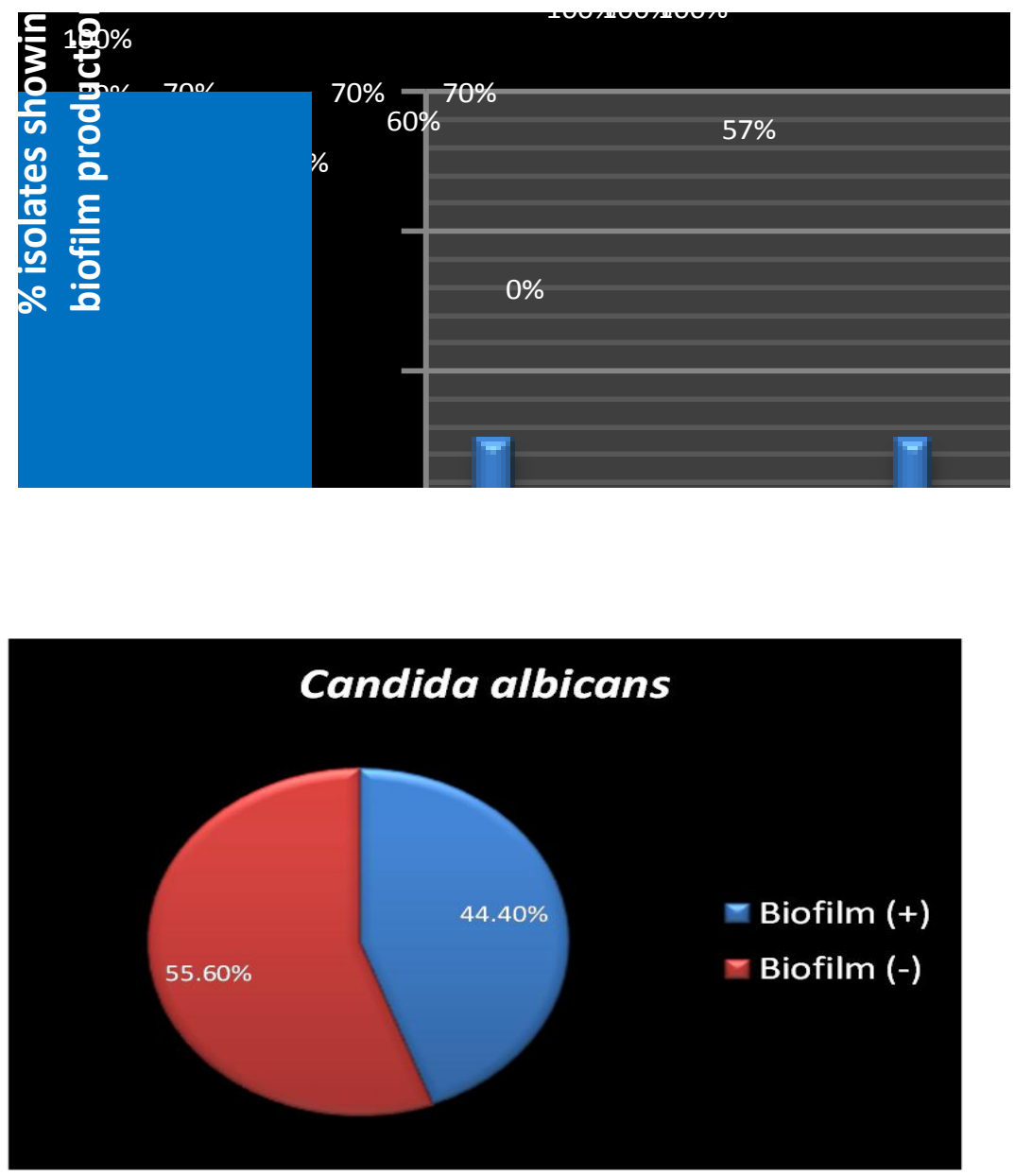

\section{Non albicans Candida}

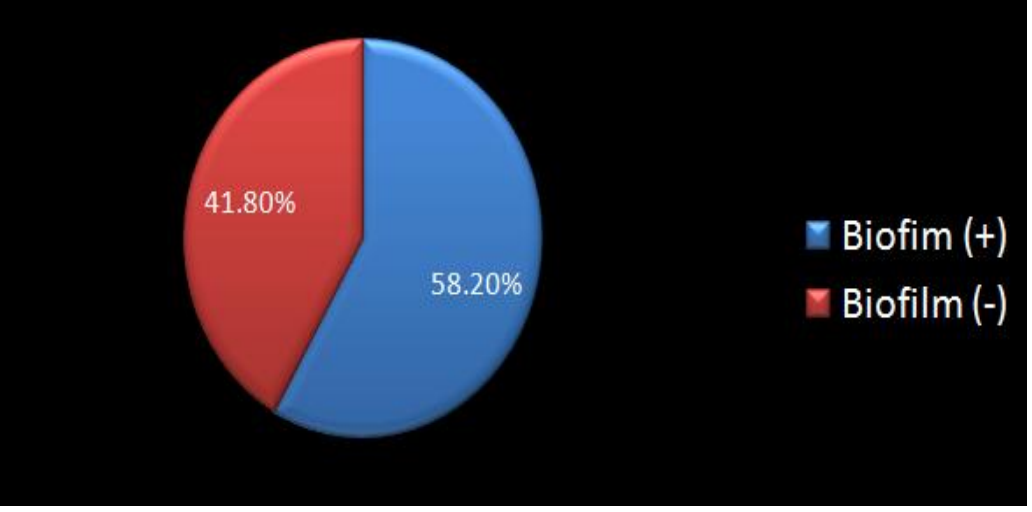


In view of various reports of infections caused by non-albicans Candida, this finding is very important (Ramage G. et al., 2001; O'Toole G.A. et al., 2000). The role of Biofilm as a virulence factor should always be kept in mind if non-albicans Candida is isolated from clinical samples.

Plant oils are increasingly claimed to have broad spectrum antimicrobial activity. Antifungal efficacy of Coconut oil and Eucalyptus oil was also tested in this study against all Candida isolates. Selected oils have been suggested to have potent antimicrobial activity against skin infection, insect bites, chicken pox, colds, flu, asthma, bronchitis and pneumonia, probably due to their phenolic, alcoholic and trepenoid constituents (Salari M.H. et al., 2006; Lu X.Q. et al., 2004; Juergens U.R. et al., 2003; Juergens et al., 2004; Serafino A. et al., 2008; Göbel H. et al., 1994; Hong et al., 1991; Hideki N et al., 2008).

The disc diffusion assay is a standard method widely used for the rapid screening of natural products for antifungal activity. Plant oils were screened using this very convenient assay method. The results indicate that caution is needed, since different oils have different diffusion rates on agar plates. This may contribute to variations in the size of the inhibitory zones, leading to erroneous conclusions regarding their antifungal activity. Hence this assay was run in triplicate and the average zone size was considered for interpretation. Both the oils were effective and showed antiCandida activity at low concentrations, however Eucalyptus oil showed better antifungal activity as compared to coconut oil.

When coconut oil was tested against all Candida albicans isolates, the sensitivity of biofilm non producers was higher in comparison to biofilm producers as shown in Table 6. Also, in the case of Eucalyptus oil $100 \%$ sensitivity was obtained against non biofilm producers and 50\% against biofilm producers. This has been shown in Table 7. When coconut oil was tested against all non albicans Candida isolates, the sensitivity of biofilm non producers was twice as compared to biofilm producers. Whereas, in the case of Eucalyptus oil $73.7 \%$ sensitivity was obtained against non biofilm producers and $39.6 \%$ against biofilm producers. The sensitivity and resistance pattern have been shown in Table 8 and 9 .

This indicates that Biofilm producers resist the antifungal activity of plant oils. This can be due to failure of oil to penetrate the full depth of the Biofilm.

In a study conducted by Vishnu Agarwal et al (2007), 30 different plant oils were tested against Candida albicans. Majority of oils in their study showed anti-Candida activity. However, they have not tested the effect on biofilm producers.

The results presented in this study clearly demonstrate the antifungal potential of the selected plant oils. Eucalyptus oil may be used as anti-biofilm agent at low concentrations. Both oils used in this study have a long history of use in food, confectionary and as components of perfume.

However, before they are considered for use as topical preparations, a careful exploration of their undesirable effects needs to be undertaken.

In conclusion, Biofilm is one of the known virulence factors of Candida, an important pathogen and commensal. Early detection of Biofilm production may be useful for clinical decision because of its suggestive property for potential pathogenic capacity of Candida isolates. 
The results presented in this study further emphasize the role of biofilm in Candida albicans as well as non-albicans Candida as a virulence factor.

It also encourages the need for further examination of the efficacy of plant oils against other forms of systemic and superficial fungal infections.

It also encourages the exploration of their broad spectrum effects against other pathogenic manifestations, including malignancies, in the coming years.

\section{References}

Brauer, A.W., Shervis, M.M., Truck, N. 1996. Antibiotic susceptibility testing by a standardized sample disc method. J. Chem. Pathology., 45: 493-496.

Christensen, G.D., Bisno, A.L., Simpsom, W.A., Beachey, E.H. 1982. Adherence of slime producing strains of

Christensen, G.D., Simpson, W.A., Yonger, J.J., Baddor, L.M., Barrett, F.F., Melton, D.M., Beachey, E.H. 1985. Adherence of coagulase-negative staphylococci to plastic tissue culture plates: a quantitative model for the adherence of staphylococci to medical devices. J. Clin. Microbiol., 22: 9961006.

Crump, J.A., Collignon, P.J. 2000. Intravascular catheter-associated infections. Eur. J. Clin. Microbiol. Infect. Dis., 19: 1-8.

Dag, I., Kiraz, N., Oz, Y. 2010. Evaluation of different detection methods of biofilm formation in clinical Candida isolates. Afr. J. Microbiology Res., 4(24): 2763-2768.

Dhanasekaran, D., Thajuddin, N., Rahhmi, Deepika, T.L., Gunasekaran, M. 2014. Human dental biofilm: Screening, characterization, in vitro biofilm formation and antifungal resistance of Candida spp. Saudi J. Dent. Res., 5(1): 55-70.

Douglas, L.J. 2002. Medical importance of biofilms in Candida infections. Rev. Iberoam. Micol., 19: 139-143.

Epka, O.D., Ebana, R.U.B. 1996. Comparative studies of Mmanyanga, Palm and Coconut oils; Anti-microbial Effects of the oils and their metallic soaps on some bacteria and fungi. Global J. Pure Appl. Sci., 1(2): 155163.

Freeman, D.J., Falkner, F.R., Keane, C.T. 1989. New method for detecting slime production by coagulase-negative staphylococci. J. Clin. Pathol., 42: 872-874.

Göbel, H., Schmidt, G., Soyka, D. 1994. Effect of peppermint and eucalyptus oil preparations on neurophysiological and experimental algesimetric headache parameters. Cephalalgia: Int. J. Headache, 14(3): 228-34.

Golia, S., Hittinahalli, V., Sangeetha, K. T., Vasudha, C.L. Study of biofilm formation as a virulence marker in Candida species isolated from various clinical specimens.

Hideki, N. et al. 2008. Effect of eucalyptus extract chewing gum on periodontal health: a double-masked, randomized trial. J. Periodontol., 79(8): 1378-85.

Hong, C.Z., Shellock, F.G. 1991. Effects of a topically applied counterirritant (Eucalyptamint) on cutaneous blood flow and on skin and muscle temperatures. A placebo-controlled study. Amer. J. Physi. Med. rehabilitation / Asso. Academic Physiatrists, 70(1): 29-33.

Juergens, U.R., Dethlefsen, U., Steinkamp, G., Gillissen, A., Repges, R., Vetter, H. 2003. Anti-inflammatory activity of 1.8-cineol (eucalyptol) in bronchial asthma: A double-blind placebo- 
controlled trial. Resp. Med., 97 (3): $250-6$.

Juergens, Uwe, R., Engelen, Tanja, Racké, Kurt, Stöber, Meinolf, Gillissen, Adrian, Vetter, Hans. 2004. Inhibitory activity of 1,8-cineol (eucalyptol) on cytokine production in cultured human lymphocytes and monocytes. Pulmonary PharmacoL. Therapeutics, 17(5): 281-7.

Kourkoumpetis, Themistoklis, K., et al. 2011. The effect of cumulative length of hospital stay on the antifungal resistance of Candida strains isolated from critically ill surgical patients. Mycopathologia., 171(2): 85-91.

Lu, X.Q., Tang, F.D., Wang, Y., Zhao, T., Bian, R.L. 2004. Effect of Eucalyptus globulus oil on lipopolysaccharideinduced chronic bronchitis and mucin hypersecretion in rats. Zhongguo Zhong yao za zhi $=$ Zhongguo zhongyao zazhi $=$ China $J$. Chinese Materia Medica., 29(2): 168-71.

Maki, D.G., Stolz, S.M., Wheeler, S., Mermel, L.A. 1997. Prevention of central venous catheter-related bloodstream infection by use of an antiseptic-impregnated catheter: a randomized, controlled trial. Ann. Intern. Med., 127: 257-66.

Manolakaki, D., Velmahos, G., Kourkoumpetis, T., Chang, Y., Alam, H .B., De Moya, et al. 2002. Candida infection and colonization among trauma patients. Virulence., 1(5): 367375.

Ogbolu, D.O., Oni, A.A., Daini, O.A., Oloko, A.P. 2007. In vitro antimicrobial properties of coconut oil on Candida species in Ibadan, Nigeria. J. Med. Food., 10(2): 384-7.

Ogbolu, D.O., Oni, A.A., Daini, O.A., Oloko, A.P. 2009. Susceptibility of Candida isolates to virgin coconut oil and fluconazole. J. Med. Food., 10(2).

O'Toole, G.A., Kaplan, H.B., Kolter, R. 2000. Biofilm formation as microbial development. Annu. Rev. Microbiol., 54: 49-79.

Ramage, G., Vande, W.K., Wickes, B.L., Lopez, J.L. 2001. Biofilm formation by Candida dubliniensis. J. Clin. Microbiol., 39: 3234-40.

Salari, M.H., Amine, G., Shirazi, M.H., Hafezi, R., Mohammadypour, M. 2006. Antibacterial effects of Eucalyptus globulus leaf extract on pathogenic bacteria isolated from specimens of patients with respiratory tract disorders. Clin. Microbiol. Infec., 12(2): 194-6.

Serafino, A., Vallebona, S., Andreola, P.S., Zonfrillo, F., Mercuri, M., Federici, L., Rasi, M., Garaci, G., Pierimarchi, E.P. 2008. Stimulatory effect of Eucalyptus essential oil on innate cell-mediated immune response. BMC Immunol., 9: 17.

Staphylococcus epidermidis to smooth surfaces. Infect Immun., 37: 318-326.

\section{How to cite this article:}

M. Bansal, S.A., Samant, S. Singh and Talukdar, A. 2016. Phenotypic Detection of Biofilms in Candida Species Isolated from Various Clinical Samples. Int.J.Curr.Microbiol.App.Sci. 5(3): 47-56. doi: http://dx.doi.org/10.20546/ijcmas.2016.503.007 